\title{
Ideenmanagement im Rahmen von organisatorischem Wandel
}

\section{Eine Triangulation der Ergebnisse der Aktionsforschung zum Ideenwettbewerb bei BMW Financial Services}

\author{
Daniel Klein, Ulrike Lechner \\ Institut für Angewandte Informatik, \\ Universität der Bundeswehr München
}

\section{Einleitung}

Den Begriffen organisatorischer Wandel und Veränderungsmanagement (Change Management) haftet eher eine negative Note an, als dass sie von vorneherein mit vielversprechenden Aussichten hinsichtlich der zukünftigen Unternehmensentwicklung in Verbindung gebracht werden (Cameron und Quinn 2006). Das gilt vor allem für den Standpunkt der Mitarbeiter. Organisatorische Veränderungen, die für den Erhalt der Wettbewerbsfähigkeit notwendig sind (Bauer-Harz 1995), gehen oft mit Personalabbau und deshalb mit Verunsicherung in der Unternehmenskultur einher.

Innovationsmanagement und artverwandte Konzepte und Instrumente wie Ideenmanagement oder Ideenwettbewerbe rufen in Wirtschaft und Gesellschaft dagegen überwiegend positive Assoziationen hervor. Der Begriff Innovation steht für Attribute wie Wachstum, Weiterentwicklung und technischen Fortschritt (Hauschildt 1997). Folglich werden Maßnahmen in Unternehmen, die mit Innovationen und Ideen zu tun haben, wahrscheinlich nicht auf kulturell bedingte Widerstände stoßen - wenn man von Veränderungen, die explizit durch Innovationen hervorgerufen werden, einmal absieht. Wir folgen dem Innovationsbegriff von Damanpour (1991), der den Fokus auf den Neuigkeitscharakter für die adaptierende Organisation legt und zudem der weit ausgelegten Definition von Rogers (2003), die neue Ideen für den Empfänger mit einschließt, ohne direkt auf die Verwirklichung dieser Ideen abzuzielen.

In unserer Forschung geht es um die gezielte Verwendung von Ideenmanagement im Rahmen von organisatorischem Wandel. Dabei konzentrieren wir uns auf die Nutzung eines Ideenwettbewerbs zur Unterstützung der Ziele von Change Management. In unserer vorangehenden Arbeit (Klein und Lechner 2009a) analy- 
sierten wir die Fähigkeiten des Ideenwettbewerbs, den Wandel auf der kulturellen Seite positiv zu beeinflussen. Zudem untersuchten wir verschiedene Aspekte bezüglich der Schaffung von Ideen seitens der Mitarbeiter und betrachteten zudem die Motivation der Teilnahme (Klein und Lechner 2009b). Einen Teil dieser Ergebnisse setzen wir in diesem Papier in einen neuen Bezug und beleuchten Sie vor dem Hintergrund zweier anderer, unterschiedlicher Datenquellen.

Die von uns genutzte Methodik ist die Aktionsforschung. Die Voraussetzungen und gleichzeitig der Bedarf für diesen Forschungsansatz entstanden durch die Tatsache, dass einer der Autoren bei der BMW Group Financial Services Deutschland (BMW FS) arbeitet, wo die konstruierte Kombination aus Ideenmanagement und organisatorischem Wandel in einem Projekt zu finden war. Der bisherige Teil unserer Forschung resultiert in der Bildung von Thesen (Klein und Lechner 2009a; Klein und Lechner 2009b). In der vorliegenden Arbeit triangulieren wir die entstandenen Thesen in ihrer Bedeutung und betrachten sie explanativ.

Im nächsten Abschnitt gehen wir näher auf die Situation bei BMW FS und auf die Voraussetzungen ein, die dieses spezifische Forschungsprojekt ermöglichten. In Abschnitt drei behandeln wir die methodische Vorgehensweise. Im vierten Abschnitt präsentieren und diskutieren wir unsere bisherigen Forschungsergebnisse und treffen die Auswahl der zu triangulierenden Thesen. Abschnitt fünf enthält die Ergebnisse dieses Papiers und der letzte Abschnitt liefert eine Zusammenfassung unserer Resultate.

\section{Forschungshintergrund bei BMW Financial Services}

Im Jahr 2007 wurde bei BMW FS ein Veränderungsprojekt ins Leben gerufen, das durch Maßnahmen eines organisatorischen Wandels einen vorgegebenen Ergebnisbeitrag bis zum Jahr 2010 sichern sollte. Das Change Management hatte demnach die Aufgabe, den Wandel zu kommunizieren und die Mitarbeiter in das Geschehen einzubinden, um die gesetzten finanziellen und strukturellen Ziele auch tatsächlich erreichen zu können. Diese pragmatische und nachhaltige Sichtweise orientiert sich inhaltlich an der gängigen Meinung der Literatur, wie z. B. bei Schreiber (2001) oder Champy und Nohria (1999) zu sehen ist.

Eine Säule des Change Management war das Thema „Innovationsmanagement" - hauptsächlich in der Bedeutung Ideen zu schaffen und zu sammeln, die das Erreichen der gesetzten Ziele unterstützen sollten. Durch den hohen Ergebnisdruck des Change Projekts und weitere Einschränkungen wie sehr knappes Budget, minimale personale Kapazitäten und kaum Zeit für Konzeption und Planung, einigte man sich auf einen Ideenwettbewerb als bestmögliche Lösung. Dabei ging es bei dem Wettbewerb inhaltlich um das Sammeln von Mitarbeiterideen, um Erträge, Kosten- und Risikostrukturen zu optimieren, analog der Ziele des Change Projekts. Kulturell sollte der Wettbewerb genutzt werden, den organisatorischen Wandel zu kommunizieren und die Mitarbeiter einzubinden. Gemäß Flynn et al. 
(2003) ist eine klare strategische Richtung Voraussetzung, um überhaupt effektiv Innovationen zu erschaffen und zu managen.

Bei der ad-hoc notwendigen systemischen Umsetzung entschied man sich für die Nutzung und Modifikation des existierenden Wissensmanagementsystems von BMW FS. Neben Zeit- und Kostenersparnissen brachte das den Vorteil, dass die Wissensplattform im Unternehmen bereits bekannt war. Dem Change Management stand somit zugleich eine bilaterale Kommunikationsplattform zur Verfügung.

\section{Methodik}

\subsection{Ansatz der Aktionsforschung}

In Übereinstimmung mit dem Bedarf an spezifischem Wissen, an tiefen Einblicken in die Unternehmung und mit unserem Bestreben, die Vorgänge selbst maßgeblich beeinflussen zu können, war Aktionsforschung der geeignete Ansatz für unsere Untersuchung. Einer der Autoren war verantwortlich für Konzept und Design des Wettbewerbs inklusive der Systemlösung. Zudem half er bei der Implementierung des Wettbewerbs und unterstützte das Change Management bei der Administration des Events. Im Anschluss an den Ideenwettbewerb untersuchte der Forscher die Einstellung der Mitarbeiter gegenüber dem Wettbewerb mit einer Umfrage. Zusätzlich führte er Interviews mit zwei Experten, die hinter den Kulissen des Wettbewerbs gearbeitet hatten und begleitete eine Umfrage im Sinne eines Change Monitorings, die bei Geschäftsleitung, Führungskräften und Mitarbeitern durchgeführt wurde.

Die eben beschriebene Situation beinhaltet mit die wichtigsten Charakteristika von Aktionsforschung nach Baskerville (1999), an orientation towards change and action, a practical problem focus, an organic process involving systematic and sometimes iterative stages, and collaboration among participants. Gemäß Gilmore et al. (1986) war unser Ziel: to contribute both to the practical concerns of people in an immediate problematic situation and to further the goals of social science simultaneously. Die Problemsituation bei BMW FS bestand darin, innerhalb kurzer Zeit einen Ideenwettbewerb auf die Beine zu stellen, um Inhalte für das Veränderungsprojekt zu produzieren, die Kommunikation des Wandels zu ermöglichen und die Mitarbeiter in das Unternehmensgeschehen zu involvieren. Der wissenschaftliche Beitrag unserer Forschung ist ein besseres Verständnis davon, wie sich ein Ideenwettbewerb - und im weiteren Sinne Ideenmanagement im Allgemeinen - im Rahmen eines organisatorischen Wandels auf Unternehmenskultur, Mitarbeiter und deren Kreativität auswirkt.

Die Daten, auf welchen unsere Forschung basiert, entstammen auch der Dokumentation des Projekts und den entstandenen Diskrepanzen zwischen theoretischem Konzept und tatsächlicher Abwicklung des Wettbewerbs. Der Großteil der 
gezielt erhobenen Daten als wichtigstes Fundament unserer Ergebnisse stammt jedoch aus der Mitarbeiterumfrage, den Experteninterviews und dem Change Monitoring.

\subsection{Problemlösung und Forschungsinteresse}

Durch die oftmals turbulenten und zeitkritischen Rahmenbedingungen innerhalb des Projekts kam es zu zahlreichen Überlappungen bei der Aufstellung des klassischen Forschungszyklus der Aktionsforschung nach Baskerville (1999). Deshalb orientieren wir uns an McKay und Marshall (2001), die eine Auftrennung der Aktionsforschung in einen Problemlösungskreislauf und einen Forschungskreislauf vorschlagen. Dabei konzentrieren sich die beiden Zyklen je exklusiv auf Problemlösung und das Erlangen von Forschungsergebnissen. Während wir beim Problemlösungskreislauf nur eine Schleife fahren, durchlaufen wir beim Forschungskreislauf nach der Vorgabe von Baskerville und Myers (2004) und O’Brien (2001) mehrere Iterationen. In unserer bisherigen Arbeit finden sich detaillierte Beschreibungen von Problemlösungskreislauf und Forschungskreislauf (Klein und Lechner 2009a; Klein und Lechner 2009b). In diesem Papier begnügen wir uns mit einer kurzen Beschreibung beider Kreisläufe und konzentrieren uns auf die Triangulation der erlangten Ergebnisse. Abbildung 1 zeigt den Problemlösungszyklus.

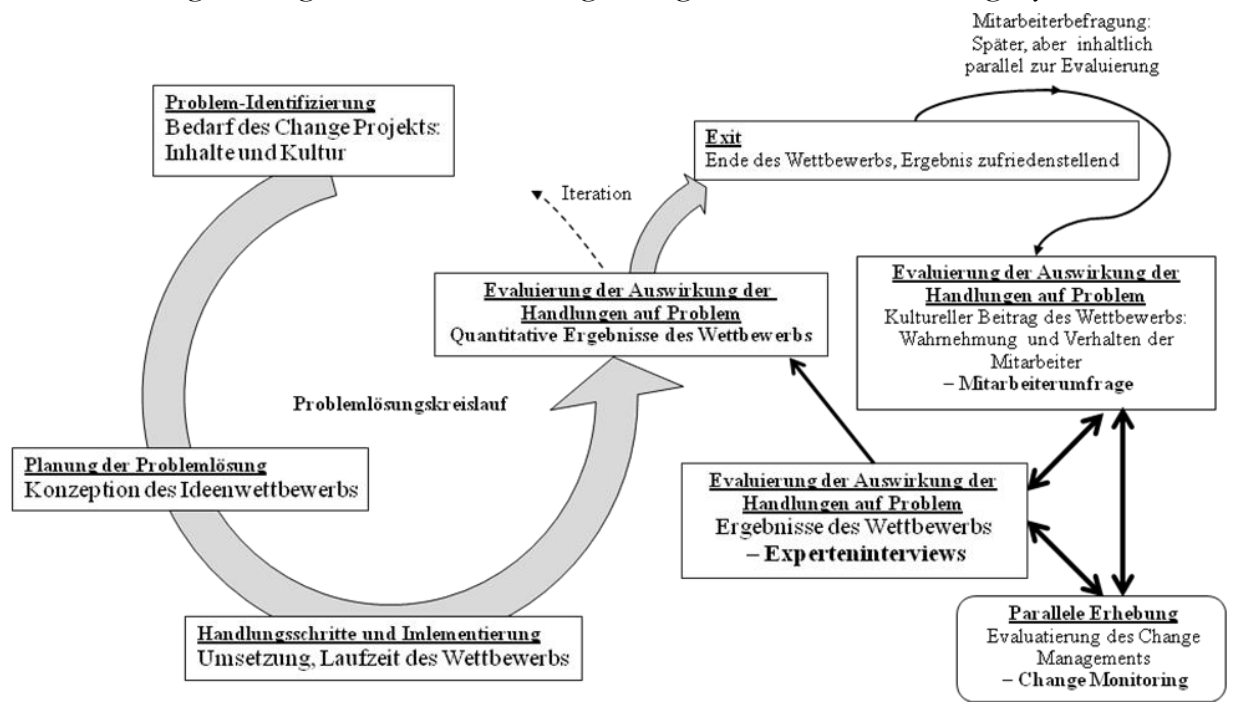

\section{Abbildung 1: Der Problemlösungskreislauf}

Der Bedarf einer geeigneten Initiative, um die Ziele des Change Management bei geringer Ressourcenbelastung zu unterstützen, ist das Ergebnis der ProblemIdentifizierung am Anfang des Problemlösungszyklus. Die Planung der Problemlösung brachte das Konzept des kurzfristigen, dreimonatigen Ideenwettbewerbs hervor, einen Aufruf an alle Mitarbeiter, ihre Ideen bereitzustellen und das Unternehmen 
zu unterstützen. Die besten Ideen würden in einem Ranking veröffentlicht und nach Ende des Wettbewerbs mit Sachpreisen prämiert. Innerhalb weniger Wochen wurde mit den notwendigen Handlungsschritten begonnen, um den Wettbewerb umzusetzen. Wichtige Punkte hierbei waren z. B. die Kommunikation des Wettbewerbs, die Modifikation der Wissensmanagementplattform und die Organisation der Prämien. Die eigentliche Implementierung des Wettbewerbs bedeutete u. a. fortschreitende Kommunikation, die Administration des IT-Systems, die Bewertung der Ideen und die zeremonielle Übergabe der Preise zum Ende der Veranstaltung.

Die Evaluierung der Auswirkungen der Handlungen auf das Problem war gleichbedeutend mit der Evaluierung des Ideenwettbewerbs an sich. Einerseits ging es dabei um quantitative Ergebnisse, wie Teilnahmezahlen und finanzielle Potentiale, andererseits um den kulturellen Beitrag des Wettbewerbs zur Unterstützung des organisatorischen Wandels. Die Ergebnisse der kulturellen Analyse stammen aus der Mitarbeiterumfrage, die im Anschluss an den Wettbewerb durchgeführt wurde. Der „Exit“ aus dem Problemlösungskreislauf erfolgte mit der Mitarbeiterumfrage und mit der Befragung der Experten. Aufgrund der zufriedenstellenden Ergebnisse und der aktuellen Unternehmenssituation, gab es keinerlei Bedarf für eine Iteration.

Die wissenschaftliche Auswertung des kulturellen Beitrags entspricht den drei Zyklen unseres Forschungskreislaufs. Der erste Zyklus brachte unser spezifisches Forschungsinteresse durch die Planung und Konzeption des Ideenwettbewerbs hervor. Im Zuge der Implementierung und Laufzeit des Wettbewerbs zeichneten sich im Rahmen des zweiten Zyklus unsere Forschungsfragen ab, die sich u. a. mit dem Teilnahmeverhalten der Mitarbeiter und deren Auffassung des Wettbewerbs, sowie der Schaffung von Ideen und der Rolle des Anreiz-Systems befassen. Im Mittelpunkt des dritten Forschungszyklus steht die Mitarbeiterumfrage an die gesamte Belegschaft. Die auf deren Basis gebildeten Thesen setzen sich mit der Problematik der Forschungsfragen auseinander und sind das Resultat unserer bisherigen Forschungsarbeit.

\subsection{Experteninterviews und Change Monitoring}

Neben der Mitarbeiterumfrage wurden im Anschluss an den Ideenwettbewerb zwei Experten befragt, die sowohl Mitglieder des Change Management Teams waren, als auch die durch die Umsetzung des Wettbewerbs entstandene Hauptarbeitslast getragen hatten. Die ca. eineinhalbstündigen Interviews spiegeln die Sicht von hinter den Kulissen des Wettbewerbs wider und fanden in etwa zeitgleich mit der Mitarbeiterumfrage statt. Infolgedessen sind die Interviews von den Ergebnissen der Umfrage nicht beeinflusst. Die beiden Befragungen sind Teil der Evaluierung des Projekts und sollten neben einer internen Beurteilung der Abläufe des Wettbewerbs den späteren Vergleich zu den Aussagen der Mitarbeiter ermöglichen.

Zudem führte das Change Management selbst eine stichprobenartige Befragung von Geschäftsleitung, mittlerem Management und Mitarbeitern durch, um 
den Status und den Erfolg des organisatorischen Wandels über die Hierarchieebenen hinweg zu messen. Innerhalb dieses Change Monitorings gibt es Inhalte mit Relevanz für die Auswertung des Ideenwettbewerbs. In Abschnitt fünf werden die Ergebnisse von Experteninterviews und Change Monitoring untersucht und in Relation zu den bisherigen Forschungsergebnissen gestellt.

\section{Ergebnisse des Ideenwettbewerbs und bisherige Thesen}

Aus der Warte des Unternehmens war der Ideenwettbewerb in jeder Hinsicht ein Erfolg. Es gelang sowohl, das Change Projekt über den Wettbewerb positiv zu kommunizieren, die Mitarbeiter in das Geschehen einzubinden und damit kulturelle Barrieren abzubauen, als auch Inhalte zur Unterstützung der Veränderungsziele zu erheben. Während der ungefähr dreimonatigen Laufzeit des Wettbewerbs wurden von 141 Mitarbeitern 330 Ideen eingestellt. Das entspricht einer Teilnahmequote von etwa $12 \%$, gemessen an der ganzen Belegschaft. Die Ideenrücklaufquote von 28\% nähert sich stark dem von Wördenweber und Wickord (2004) prognostizierten Wert für unternehmensinterne Ideenwettbewerbe. Das größte Volumen an eingehenden Ideen wurde mit 31 Vorschlägen am letzten Tag des Wettbewerbs gemessen. Als scheinbar natürliches menschliches Phänomen bezüglich Deadlines ist dies im Sinne der Aussagen von Elam und Mead (1987) bezüglich Stress als Treiber für Kreativität interpretierbar. Das mit 24 Ideen zweitgrößte Volumen trat etwa zur Halbzeit direkt als Reaktion auf eine Erinnerungsemail des Change Management Teams auf. Zusammen mit einem zweiten Spitzenwert dieser Natur spricht das für die Wirksamkeit und Notwendigkeit einer adäquaten Kommunikation zum Erreichen einer hohe Teilnahmequote, analog zu den Bemerkungen von Leach et al. (2006) zur internen Öffentlichkeit im Ideenmanagement.

Die sieben besten Ideen hatten ein kalkuliertes finanzielles Potential von über dem 90fachen der ursprünglichen Kosten des gesamten Ideenwettbewerbs. Die Bewertung der Ideen orientierte sich stark an den Zielen des Change Projekts. Dabei wurde der abzusehende Kapitalwert des Ergebnisbeitrags der Ideen über die nächsten drei Jahre ermittelt und mit einem Faktor der Eintrittswahrscheinlichkeit multipliziert. Die entstandenen Kosten setzen sich maßgeblich aus den Prämien und den Maßnahmen der IT- Modifikationen zusammen, personelle Aufwände wurden bei der Betrachtung außen vor gelassen. Es bleibt zu erwähnen, dass die eingegangenen Innovationsvorschläge überwiegend inkrementeller Natur waren und häufig aus dem eigenen Arbeitsumfeld der Ideengeber stammten. Laut Vandenbosch et al. (2006) werden Ideen ständig generiert, nur das Maß der Kreativität sei dabei in Frage zu stellen.

Die Mitarbeiterumfrage im Anschluss an den Wettbewerb richtete sich an alle Mitarbeiter von BMW FS. Der Aufruf zur Teilnahme am anonymen OnlineFragebogen erfolgte via eMail durch ein Mitglied der Geschäftsführung. Insgesamt beteiligten sich 317 Mitarbeiter an der Umfrage, davon beantworteten 221 alle 
maximal 33 Fragen. Um die Beeinflussung der Probe durch Selbstselektion zu vermeiden, wurden die Fragen im Nachhinein für Ideengeber und NichtIdeengeber getrennt ausgewertet. Die prozentuale Beteiligung der Ideengeber war dabei wie zu erwarten höher und zeigte über den Verlauf der Fragen weniger Degression.

Unsere bisherigen Forschungsergebnisse basieren maßgeblich auf den durch die Mitarbeiterumfrage erhobenen Daten. Wir bildeten Thesen zur Wirkungsweise von Ideenwettbewerben im Rahmen von organisatorischem Wandel und zu unternehmensinternen Ideenwettbewerben im Allgemeinen.

Die Thesen besagen, dass ein Ideenwettbewerb trotz Einbindung in die kontroverse Change-Situation weiterhin als positives Ereignis von den Mitarbeitern erlebt wird und sich in der Konsequenz auch positiv auf die Wahrnehmung des Change Management auswirkt. Während dies auch für passive, nicht-teilnehmende Mitarbeiter gilt, stellen wir bei Ideengebern eine überproportional positive Wahrnehmung fest. Weiterhin fühlen sich die Mitarbeiter durch den Ideenwettbewerb mehr in das Unternehmensgeschehen eingebunden. Dies geht einher mit der Aussage von Klotz (1988) zu Ideenmanagement im Allgemeinen und trifft sogar für Mitarbeiter zu, die nicht am Wettbewerb teilgenommen haben. Im Kontext des organisatorischen Wandels proklamieren wir „Um das Unternehmen zu unterstützen" als wichtigsten Grund der Teilnahme am Wettbewerb. Weiterhin arbeiten wir die nicht vorhandene Bedeutung von Prämien als notwendige Anreize heraus - im Widerspruch zur Bedeutung der Höhe von Prämien im Vorschlagswesen bei Ideen um Kosten zu sparen, nach Rapp und Eklund (2002) und zur Bedeutung von Anreizsystemen im Allgemeinen (z. B. Witt 2000, Thom und Etienne 1999). Wir erkennen den Faktor Zeit im Sinne von verfügbarer Zeit für den Mitarbeiter, um Ideen zu erdenken und einzustellen als kritischen Erfolgsfaktor für eine hohe Teilnahmequote.

In den Thesen halten wir weiterhin fest, dass der Großteil der Ideen aus dem eigentlichen Aufgabenbereich der Ideengeber stammt. Für die meisten dieser Ideen wurde der Wettbewerb als Gelegenheit gesehen und genutzt, sie zu platzieren. Zudem fungiert der Wettbewerb als Auslöser (Trigger) für die Schaffung von neuen Ideen. Dabei werden besonders Ideen stimuliert, die außerhalb des eigentlichen Aufgabenbereichs der Ideengeber liegen.

In diesem Papier greifen wir speziell diese Thesen wieder auf, die sich mit dem Wettbewerb im Ganzen und der Verbindung von Wettbewerb und organisatorischem Wandel befassen, liegt hier doch das Hauptaugenmerk unserer Forschung:

T1: Ein Ideenwettbewerb im Rabmen eines organisatorischen Wandels wird von den Mitarbeitern als positives Ereignis wahrgenommen und verbessert dadurch die Wabmehmung des organisatorischen $W$ andels an sich.

T2: Ein Ideenwettbewerb im Rabmen eines organisatorischen Wandels verbessert die empfundene Einbindung der Mitarbeiter in das Unternehmensgeschehen. 
T3: Für Mitarbeiter ist der Hauptgrund der Teilnahme an einem Ideenwettbewerb im Rabmen eines organisatorischen $W$ andels, das Unternehmen zu unterstützen.

T4: Ein Ideenwettbewerb im Rahmen eines organisatorischen Wandels braucht keine Prämien als maßgebliche Motivation der Teilnahme.

Im folgenden Abschnitt werden wir diese Thesen mit Hilfe der Daten aus Experteninterviews und Change Monitoring triangulieren und somit auf ihre Validität von einer internen Perspektive prüfen.

\section{Triangulation ausgewählter Ergebnisse}

Bei den beiden Experteninterviews handelt es sich um ca. eineinhalbstündige, semi-strukturierte Befragungen zweier Mitglieder des Change Management Teams. Die Interviews behandeln die Rolle der Befragten, den Ideenwettbewerb im Ganzen, die Inhalte der Ideen, die Eindrücke als Ideenbearbeiter und den Umgang mit der systemischen Lösung. Neben der freien Beantwortung der meisten Fragen waren bei einigen Punkten feste Antwortkategorien vorgegeben. Manche der Fragen ermöglichen es, die Ergebnisse der Mitarbeiterumfrage in einem anderen $\mathrm{Zu}-$ sammenhang zu beleuchten, einige Fragen wurden den Mitarbeitern in derselben Form gestellt.

Das Change Monitoring war eine strukturierte Befragung von 41 Personen aus verschiedenen Ebenen des Unternehmens. Die Fragen befassen sich mit der Transparenz der Vorgänge im Change Management und der wahrgenommenen Zielgerichtetheit und Aufgabenerfüllung der Rollenträger im Change Projekt. Es wurden per Zufall Mitarbeiter, Teamleiter, Abteilungsleiter und Mitglieder der Geschäftsleitung ausgewählt. Mitarbeiter stellten dabei mit über 50\% die Mehrheit der Befragten. Schnittmengen von hier befragten Mitarbeitern und Teilnehmern der Mitarbeiterumfrage sind möglich. Zwischen den beiden Erhebungen lagen allerdings ca. zwei Monate und sowohl Rahmen als auch Inhalte waren komplett unterschiedlich.

These T1 basiert u. a. auf der Tatsache, dass laut Mitarbeiterumfrage 52\% der Mitarbeiter dem Ideenwettbewerb offen oder positiv und ein Drittel neutral gegenüber standen. Selbst 44\% der Nicht-Ideengeber waren in ihrer Einstellung gewogener als neutral. Beim Change Monitoring wurde auf die offene Frage „Was läuft gut?" von 20 Antworten mit dem größten Anteil sieben Mal der Ideenwettbewerb genannt. Einer der Experten antwortete auf die Frage nach dem Erfolg des Wettbewerbs als Werkzeug des Change Management mit erfolgreich, der andere mit absolut erfolgreich. Dabei gewichten im Mittel beide die Kommunikation des Change Management und die Einbindung der Mitarbeiter gegenüber der Erhebung von Inhalten mit 75\% als Hauptbeitrag des Wettbewerbs. Die Erfüllung dieser Ziele spricht nach Meinung der Interviewpartner für eine breite Akzeptanz des Ideenwettbewerbs und ein reges Interesse bei den Mitarbeitern. Zudem vertraten beide 
Experten im Gespräch aufgrund ihrer persönlichen Erfahrungen vor, während und nach dem Event die Auffassung, dass der Wettbewerb insgesamt sehr gut bei den Mitarbeitern angekommen sei. T1 sieht sich somit durch zwei weitere Quellen bestätigt. Dies ist auch im Einklang mit der Meinung des Autors, der den Ideenwettbewerb als positiv wahrgenommenes Ereignis im Unternehmen betrachtet.

Wie oben erwähnt sahen die Experten den Wettbewerb als erfolgreiches bzw. absolut erfolgreiches Werkzeug zur Zielerreichung des Change Projekts an. Auf die direkte Frage, wie gut die Einbindung der Mitarbeiter in den organisatorischen Wandel als Hauptzweck des Wettbewerbs gelungen sei, antwortete einer sehr gut gelungen, der andere gut gelungen. Das Change Monitoring stellt in dieser Richtung mehrere Fragen, die den Sachverhalt einkreisen. Der Aussage „Ich habe verstanden, dass jeder seinen Beitrag leisten muss, damit der Veränderungsprožss gelingt." stimmten 85\% zu. Dies korreliert klar mit dem Appell des zuvor abgehaltenen Ideenwettbewerbs zur Mithilfe durch das Einbringen der Ideen aller Mitarbeiter. Zudem stimmten $66 \%$ der Befragten der Aussage zu, dass eine Veränderung dringend notwendig sei, weitere $18 \%$,stimmten dem eher zu“. Diese Wahrnehmungen stehen für ein Erkennen der Notwendigkeit für Veränderungen, wie es vornehmlich durch eigene Betroffenheit und Eingebundenheit hervorgeht. Laut Mitarbeiterumfrage fühlen sich 49\% der Mitarbeiter durch den Wettbewerb mehr in das Unternehmensgeschehen eingebunden. Ein Split der Ergebnisse zeigt, dass sich 43\% der befragten Mitarbeiter, die selbst nicht aktiv am Wettbewerb teilnahmen, trotzdem mehr eingebunden fühlten. In Übereinstimmung mit Aussagen von Experteninterviews und Change Monitoring, betrachten wir T2 als bestätigt.

T3 deklariert als den Hauptgrund für die Teilnahme am Wettbewerb im Rahmen des organisatorischen Wandels den Willen, das Unternebmen zu unterstützen. 75\% von 105 befragten Ideengebern wählten diese Antwortmöglichkeit als den Hauptgrund der Teilnahme, weit vor Gelegenheit meine Idee loszunverden, Um mehr am Unternebmensgeschehen teilzubaben, Aussicht auf eine Prämie und Um Wertschätzung zu erfahren. Das korreliert mit dem oben genannten Wert aus dem Change Monitoring, wonach $85 \%$ erkennen, dass sie selbst für eine erfolgreiche Veränderung einen Beitrag leisten müssen. Wenngleich dadurch keine direkte Bestätigung von T3 vorliegt, wird das Bedürfnis der Mitarbeiter, das Unternehmen nach Möglichkeit zu unterstützen, nochmals betont.

T4 erweitert die Aussage von T3 in dem Sinne, dass sie die Notwendigkeit der Ausrichtung von Prämien in diesem Rahmen sehr relativiert darstellt. Laut Mitarbeiterumfrage wählten nur 30\% die Antwort Aussicht auf eine Prämie auf obige Frage nach dem Hauptgrund ihrer Teilnahme. Zusätzlich sagen $84 \%$ der befragten Ideengeber, Sie hätten auch ohne Aussicht auf eine Prämie teilgenommen, obwohl die Meinung von den Prämien sehr gut war. Auf die Frage nach ihrer Ansicht bzgl. der ausschlaggebenden Motivation der Mitarbeiter zur Teilnahme nannten beide Experten Aussicht auf eine Prämie. Einer der Beiden hielt zusätzlich Um Wertschätzung zu erfahren und Um mehr am Unternehmensgeschehen teilinhaben für wichtige Faktoren. Diese Betrachtung ermöglicht zwar weder das Verifizieren noch Falsifizieren von 
T3 und T4, stellt aber die Außergewöhnlichkeit der unerwarteten Ergebnisse bzgl. der Motivation der Mitarbeiter im Kontext des organisatorischen Wandels nochmals in den Vordergrund. Über das Change Monitoring ist zu T4 keine Aussage zu treffen.

\section{Zusammenfassung}

In diesem Papier beschäftigen wir uns mit den bisherigen Ergebnissen unserer Aktionsforschung bei der BMW Group Financial Services Deutschland (BMW FS) zur Nutzung eines Ideenwettbewerbs im Rahmen von organisatorischem Wandel. Wir beleuchten diese Ergebnisse aus verschiedenen Perspektiven durch das Hinzuziehen weiterer Datenquellen.

Die kulturelle Unterstützung des organisatorischen Wandels durch den Ideenwettbewerb bei BMW FS und dessen Wahrnehmung durch die Mitarbeiter wurde innerhalb unserer bisherigen Forschung maßgeblich durch eine Mitarbeiterumfrage im Anschluss an den Wettbewerb analysiert. Zusätzlich wurden zwei Mitglieder des Change Management Teams und gleichzeitige Bearbeiter des Wettbewerbs im Nachgang als Experten befragt. Diese Experteninterviews ziehen wir zusammen mit einer durch das Change Management selbst über den hierarchischen Querschnitt des Unternehmens gehaltenen Umfrage heran, um ausgewählte Thesen unserer bisherigen Forschungsresultate zu triangulieren und damit auf ihre Aussagekraft zu testen. Dabei finden wir die Aussagen zur positiven Wahrnehmung des Ideenwettbewerbs trotz direkter Einbindung in den organisatorischen Wandel bestätigt. Ebenso können wir die Aussagen zur gefühlten Mehreinbindung der Mitarbeiter in das Unternehmensgeschehen bekräftigen. Bezüglich der Motivation der Teilnahme der Mitarbeiter am Ideenwettbewerb und der Notwendigkeit von Prämien ist es uns möglich, die Besonderheiten unserer aufgestellten Thesen dahingehend zu betonen, was den Willen der Mitarbeiter, das Unternehmen zu unterstützen, als oberste Motivation betrifft.

In unserer zukünftigen Forschung werden wir unsere bisherigen Resultate durch die Analyse von vergleichbaren Sachverhalten bei anderen Unternehmen weiter explanativ beforschen. Zudem erweitern wir den Fokus auf die strategische Nutzung von Ideenmanagement im Allgemeinen und untersuchen relevante Situationen im Innovationsgeschehen bei Fällen aus der Praxis, z. B. in Form einer Multi-Case Study.

\section{Literatur}

Baskerville R (1999) Investigating Information Systems with Action Research. CAIS 2(19). 
Baskerville R, Myers M (2004) Making IS Research Relevant to Practice Foreword, Special Issue on Action Research in Information Systems. MIS QUATERLY 28(3):329-335.

Cameron K, Qinn R (2006) Diagnosing and Changing Organizational Culture. Whiley, San Francisco.

Bauer-Harz A (1995) Ethik in Unternehmen: ein Konzept für das Innovationsmanagement. Universität Mannheim.

Champy J, Nohria N (1999) Speed. Schneller als die Konkurrenz. Wiley, Metroplolitan Verlag.

Damanpour F (1991) Organizational Innovation: a Meta-Analysis of Effects of Determinants and Moderators. ACADEMY OF MANAGEMENT JOURNAL 28(3):555-590.

Elam J, Mead M (1987) Designing for creativity: Considerations for DSS development. INFORMATION AND MANAGEMENT 13 (5):215-222.

Flynn M, Dooley L, O’Sullivan D, Cormican K (2003) Idea Management for Organizational Innovation. INTERNATIONAL JOURNAL OF INNOVATION MANAGEMENT 7(4):417-442.

Gilmore T, Krantz J, Ramirez R (1986) Action Based Modes of Inquiry and the Host-Researcher Relationship. CONSULTATION 5(3):161.

Hauschildt J (1997) Innovationsmanagement. Vahlen, München.

Klein D, Lechner U (2009a) The Ideas Competition as Tool of Change

Management - Participatory Behaviour and Cultural Perception. In: Huizingh

KRE, Conn S, Torkkeli M, Bitran I (Hrsg.) Proceedings of the 20th

Conference of the International Society for Professional Innovation

Management, Vienna, June 21st-24th 2009.

Klein D, Lechner U (2009b) The Ideas Competition as Tool of Change

Management - Apects of Triggering Ideas. In: Proceedings of the Fifteenth

Americas Conference on Information Systems, San Francisco, August 6th-9th 2009. http:/ / aisel.aisnet.org/amcis $2009 / 783$

Klotz V (2002) Staff suggestion schemes.

INTERNATIONAL LABOR REVIEW 127(3):335-352.

Leach D, Stride C, Wood S (2006) The effectiveness of idea capture schemes.

INTERNATIONAL JOURNAL OF INNOVATION MANAGEMENT 10(3):325-350.

McKay J, Marshall P (2001) The dual imperatives of action research.

INFORMATION, TECHNOLOGY \& PEOPLE 14(1):46-59. 
O'Brien R (2001) Um exame da abordagem metodológica da pesquisa ação [An Overview of the Methodological Approach of Action Research], Teoria e Prática da Pesquisa Ação [Theory and Practice of Action Research]. João Pessoa, Universidade Federal da Paraíba.

Rapp C, Eklund J (2002) Sustainable development of improvement activities - the long-term operation of a suggestion scheme in a Swedish company. TOTAL QUALITY MANAGEMENT 13(7):945-969.

Rogers EM (2003) Diffusion of Innovations. The Free Press, New York.

Schreiber R (2001) Innovations-Management für soziale Organisationen: neue Strukturen entwickeln, Synergien nutzen, effiziente Organisationen aufbauen. Walhalla-Fachverlag, Regensburg.

Thom N, Etienne M (1999) Innovation und Reorganisation: organisatorische und personelle Aspekte für ein erfolgreiches Innovationsmanagement - business reengineering. ARBEITSBERICHT / INSTITUT FÜR ORGANISATION UND PERSONAL 32, Universität Bern.

Vandenbosch B, Saatcioglu A, Fay S (2006) Idea Management: A Systemic View. JOURNAL OF MANAGEMENT STUDIES 43(2):259-288.

Witt J, Bienert W (Hrsg.), Crisand E (Hrsg.)(2000) Innovatives Führen Grundlagen und Bausteine des Innovations-Managements. ARBEITSHEFTE FÜHRUNGSPSYCHOLOGIE 34, Sauer-Verlag, Heidelberg.

Wördenweber B, Wickord W (2004) Technologie- und Innovationsmanagement im Unternehmen: Methoden, Praxistipps und Softwaretools. Springer Verlag, Berlin. 